\title{
Seeing further than your nose
}

\author{
Gert van Tonder ${ }^{1}$, Daniele Zavagno ${ }^{2}$, Kenzo Sakurai ${ }^{3}$, Hiroshi Ono ${ }^{4}$ \\ ${ }^{1}$ Department of Architecture and Design, Kyoto Institute of Technology, Matsugasaki, \\ Sakyo-ku, Kyoto 606-8585, Japan; e-mail: gvtonder@yahoo.co.uk; ${ }^{2}$ Department of Psychology, \\ University of Milano-Bicocca, Milano, Italy; ${ }^{3}$ Department of Psychology, Tohoku Gakuin \\ University, Sendai, Japan; ${ }^{4}$ Department of Psychology, York University, Toronto, Canada \\ Received 18 March 2013, in revised form 5 June 2013
}

\begin{abstract}
Among monocular depth cues, ocular parallax (first described formally by Brewster in 1844) remains mostly unknown, its role in perception still not investigated scientifically. Given that every single eye movement induces ocular parallax, it is a potentially useful depth cue. This paper is an attempt to revive interest in the topic. As a monocular depth cue, ocular parallax naturally leads us to consider its benefit for a monocularly enucleated individual. Throughout history, numerous illustrious personalities coped with this fate in various ways. Here, we consider some historical insights into the visual life of the erstwhile duke of Urbino, Federico da Montefeltro (1422-1482), warlord and patron to the painter Piero della Francesca, and the Japanese warlord, Masamune Date (1567-1636), a vocal patron of exploration and the arts.
\end{abstract}

Keywords: Federico da Montefeltro, Masamune Date, monocular vision, ocular parallax

\section{Introduction}

True to the Renaissance spirit, the artists who worked in Urbino painted the likenesses of their patrons in painstaking detail. The Flemish school in the North was blossoming in the increasing realism it brought to the canvas, thanks in part to replacing the matte medium of tempera with pigments mixed into oil, and its influence was becoming evident in the portraiture of the nobility and the bourgeoisie of the Italian peninsula. Even if the realism of the likeness was becoming of prime importance, one would expect that, out of respect, and perhaps even fear, the artists would conceal physical deformities of their patrons as far as possible wherever they occurred, but how do you hide a half-missing nose?

The answer seems evident from a portrait of Federico da Montefeltro (1422-1482), duke of Urbino and patron to the painter Piero della Francesca (1416-1492). Unwilling to conduct warfare from the convenience of his armchair at home, Federico-an accomplished warlord, diplomat, and patron to the arts - was not hesitant to engage in direct combat on the battlefield. The trauma that peremptorily marked his profile, however, took place during a tournament in 1451. History records that during a jousting contest a spear striking his face dealt a heavy blow to the bridge of his nose. Bernardino Baldi (1553-1617), an early yet posthumous biographer of Federico da Montefeltro, reports the injury to the eye, caused by Guidagnolo de' Ranieri (Baldi 1824, pages 143-144), while the damage to the nose is mentioned in only general terms (note 18, page 198). Conflicting historical details abound, but it seems plausible that on direct orders from the duke himself the scar was to become a prominent feature of his profile in portrait (Roeck and Tönnesmann 2005). In fact, following the scar, the duke is depicted only in profile. Given the precision of della Francesca's rendering (figure 1a), one can assume that the scarred nose was painted in accurate detail, as it actually appeared in real life. The clean lines of the scar suggest surgical intervention on what otherwise must have been a rough gash on the nose. The scar therefore probably really looked like a peculiar square hole, through which one can see a small portion of Urbinian sky in della Francesca's painting. 


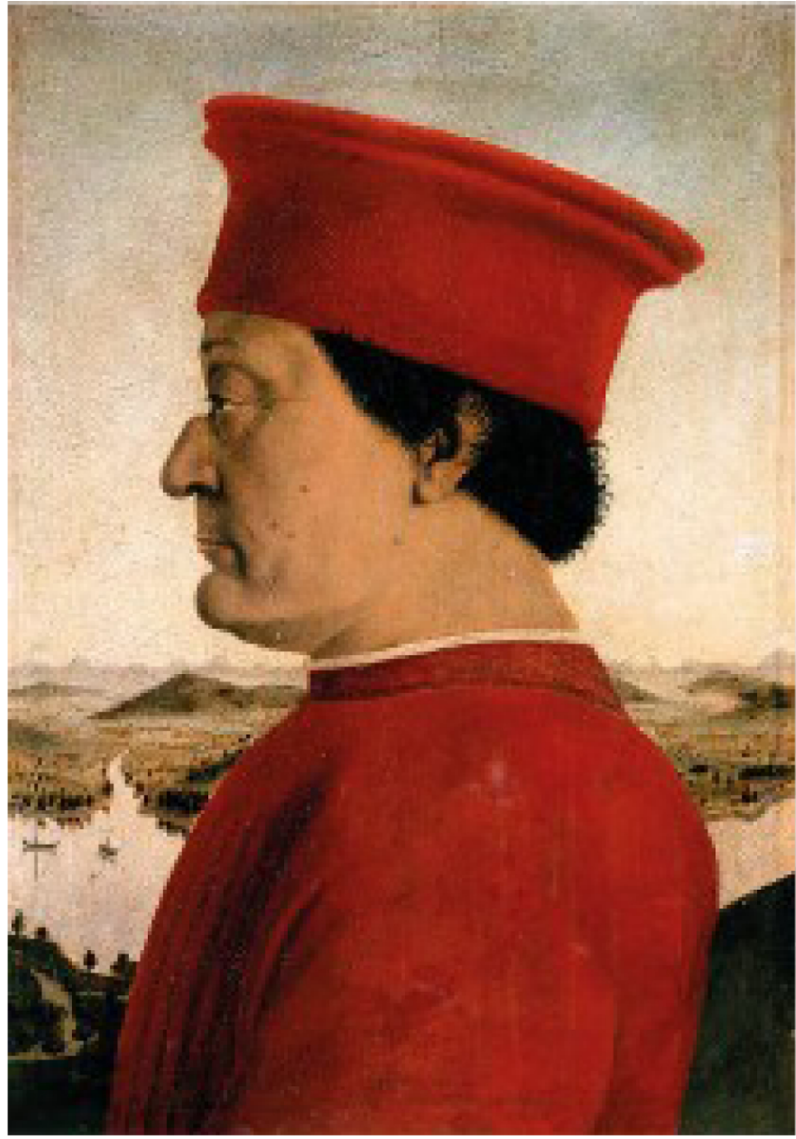

(a)

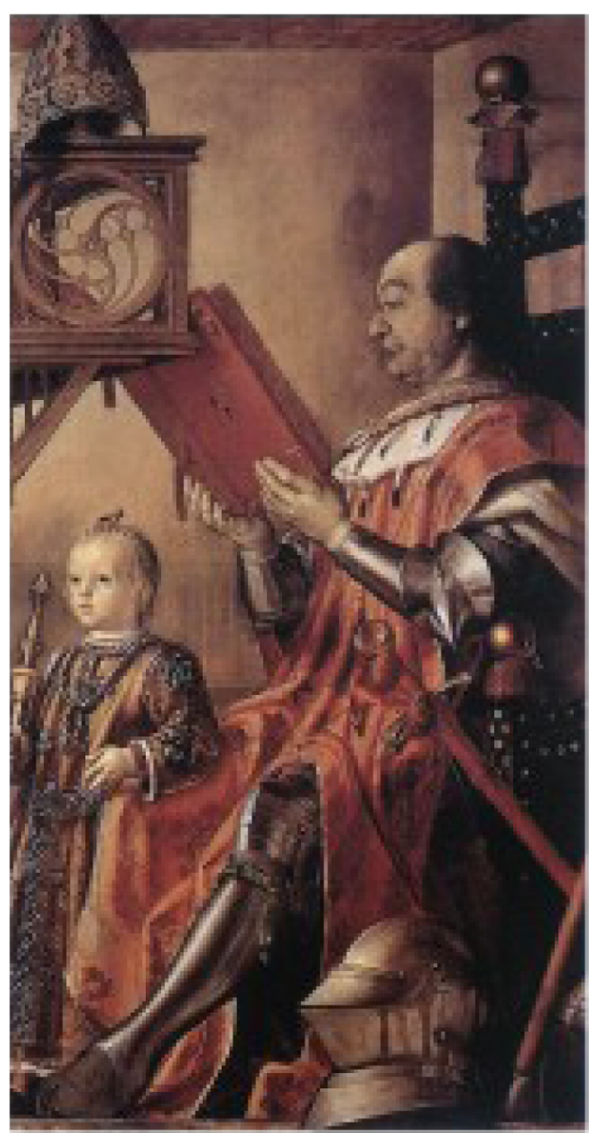

(b)

Figure 1. [In color online, see http://dx.doi.org/10.1068/p7492] Paintings of the duke of Urbino (a) by Piero della Francesca (1465), and (b) attributed to Pedro Berruguete (c. 1475-1480).

\section{Scanning the visual field with one eye}

The fact that we can see through Federico's nose should hold peculiar interest for a vision scientist. Normally, the nose protrudes into a good portion of the potential spatial overlap between the left and right eye fields, leaving one wondering why nature would deliberately obstruct what otherwise would have been a much larger region of binocular stimulation for stereopsis. Federico would have enjoyed this benefit, were it not for the unfortunate fact that the spear did not end at his nose: it struck his right eye as well, robbing him entirely of normal binocular vision. This would be the actual reason why he instructed to be painted only in profile, showing his intact left eye.

Consider, for example, his left eye field before the accident. When he looked straight ahead, the eye field would extend to its temporal maximum towards the left-restricted by only the outer limit of the retina in the eye-while the nose bridge would have protruded only slightly into the eye field on the nasal side (figure $2 b$ ). When he shifted his gaze toward the right, the ipsilateral limit would shift with the eye, while the nose bridge would have occluded a significant section of the left eye field on the nasal side (figure 2c). You can see this effect by shifting your left gaze from the far left to far right while closing the right eye. The portion of the visible eye field occluded by the nose expands as you make the shift towards the right. In fact, the apparent shape of your nose also changes, as will be explained below. This is due to ocular parallax, a forgotten and quite different cousin of the more thoroughly studied phenomenon of self-induced motion parallax. 


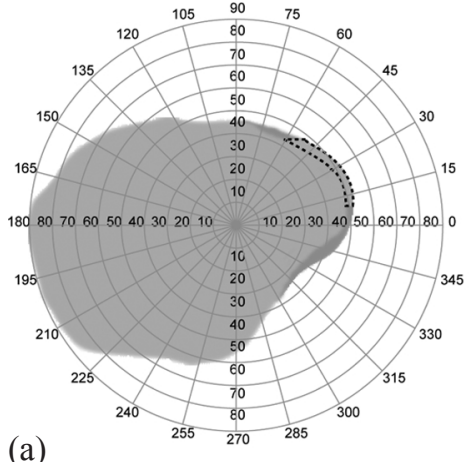

(a)

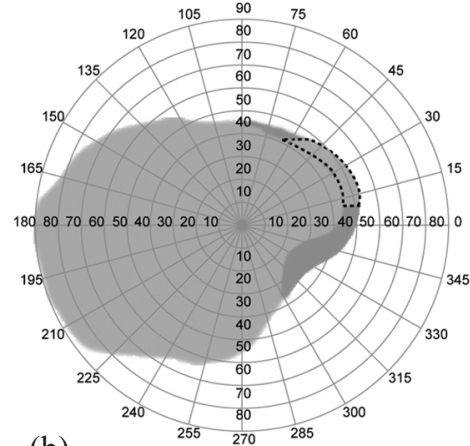

(b)
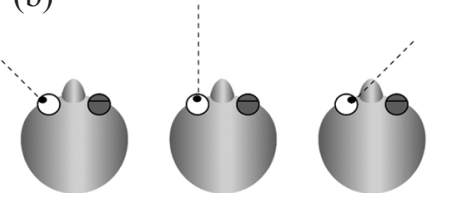

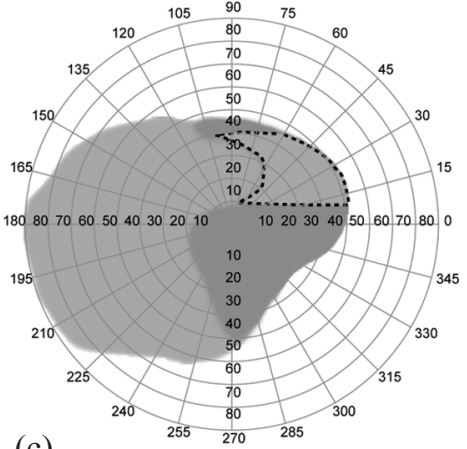

(c)

Figure 2. The left eye field, when directing the gaze towards the (a) ipsilateral, (b) central, and (c) nasal sides. The shaded regions indicate the occlusion shadow of the nose as it protrudes into the visual field. Dashed lines indicate the visual access gained by surgical removal of the upper nose bridge.

When the eye rotates in its socket, the nodal point of the ocular optics does not rotate on the center of ocular rotation, but is translated along an arc. To understand the implication of this distinction, consider three points - A, B, and C-aligned with the optical axis of one eye when you look straight ahead (figure 3, left). Needless to say, their optical projections coincide on the retina of that eye. The key observation here is that when the eye rotates their respective projections undergo relative displacements on the retina (figure 3, right) - that is, displacement parallax. If the eye did in fact rotate physically around the optical center of the
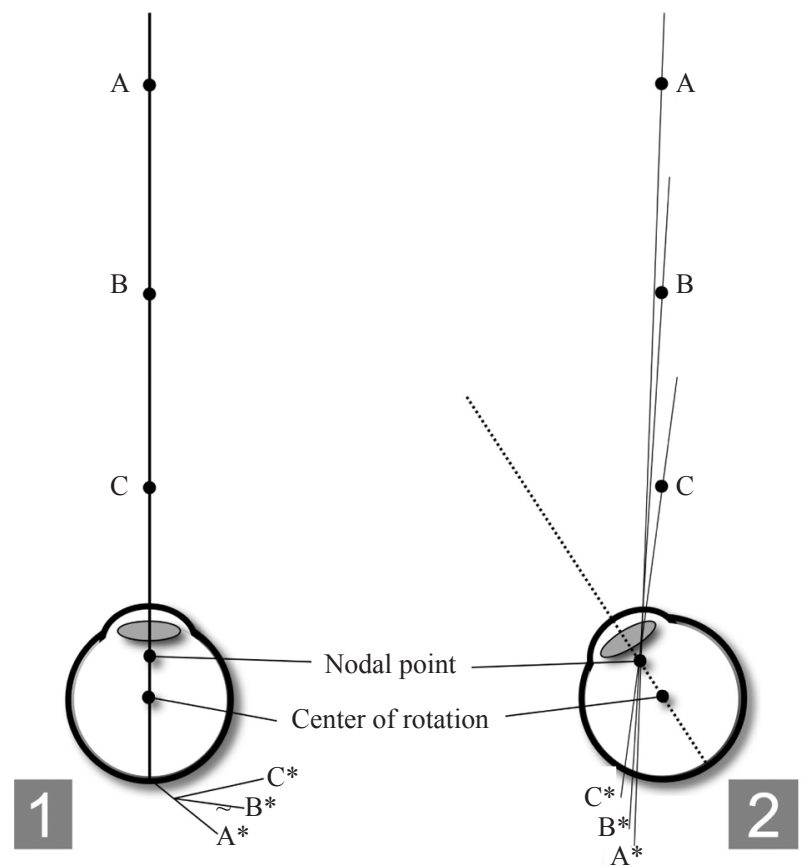

Figure 3. An approximate schematic demonstration of ocular parallax. If the retinal projections $\left(\mathrm{A}^{*}, \mathrm{~B}^{*}\right.$, $\left.\mathrm{C}^{*}\right)$ of three points $\mathrm{A}, \mathrm{B}, \mathrm{C}$ (left) coincide when viewed from a given direction of gaze, their projections will undergo relative displacement parallax (right) for any rotation of the eye - that is, their respective projections onto the retina will be displaced relative to each other, depending on their distance from the nodal point. 
cornea and lens, their retinal projections would undergo the same displacement on the retina during a gaze shift; but with the center of physical rotation placed well behind the optical center, the extent of the spatial displacement of optical projections depends on the distance between the nodal point in the eye and the object outside. The closer the object, the larger the spatial displacement of its retinal projection for a given eye rotation. This is why, looking at your nose through your left eye while slowly shifting your gaze from the far left towards the far right, you can see it swivel into view (even if blurred because it is too close to be properly focused) like a triangular swing door on a hinge (compare the dark shaded nasal projections in figures $2 \mathrm{a}, 2 \mathrm{~b}$, and $2 \mathrm{c}$ ). Owing to ocular parallax, the retinal image of the nostril moves faster and further than the projection of the nose tip during this eye movement-hence the impression that the shape of the nose itself also changes.

Brewster (1844) was among the first to describe ocular parallax (Wade 1983, pages 95-96). His description included a schematic that, in principle, is reminiscent of figure 3. Ocular parallax can be vividly demonstrated by tilting a chessboard with its pattern facing away from the nose towards the optical axis of one eye until its checkerboard pattern just becomes invisible (ie the board is aligned with the optical axis of the eye). An eye movement away from the checkerboard should vividly bring the black-and-white checker pattern of the chessboard into view, the consequence of ocular parallax between the regions nearer to and further from the observer. The effect can also be demonstrated by moving a finger from the center of the eye field towards the nasal edge of an eye field until the finger is just about to become invisible, while gazing straight ahead and keeping the contralateral eye closed (Mapp and Ono 1986). The finger, visible in the initial configuration, will disappear completely behind the nose when the gaze is suddenly directed at the finger itself. In effect, when redirecting your gaze towards the finger, the ocular nodal point translates so far toward the bridge of the nose that the nose obstructs the direct sight line between the nodal point and the finger, and hence the finger is no longer visually accessible.

Following the jousting scar, the duke would therefore have had no trouble seeing his finger throughout this demonstration of ocular parallax. With the nose bridge intact, Federico would have had to turn his head - a comparatively slow movement — to see what was lurking on the blind side of his nose. The surgical removal of the damaged nose bridge thus seems a beneficial compromise upon the loss of one eye.

As a military campaigner in the pay of the Sforza, the Aragonese, the pope, and Florence, Federico would have been greatly frustrated by the sudden loss of his right eye field. Besides being compromised on the battlefield, he would be an easier target for assassination. It seems plausible that Federico took matters into his own hands. A jousting wound would most likely appear as a visible rough gash, caused by torn cartilage and shredded bone. The clean-cut rectangular scar, rendered in detail by Piero della Francesca, is therefore considered as evidence of surgical reshaping of the nose bridge. Whether the duke acted upon his own perceptual intuition or whether he was advised by an optician of his day, this stands out as a dramatic example of mutilation in sacrifice of visual capacity. According to some accounts, after recovery the duke claimed that with the one intact eye he could see better than with a hundred eyes (Roeck and Tönnesmann 2005).

Piero della Francesca shows Federico from his left eye viewpoint (figure 1a). The slightly anterior view of the ear, and the alignment of the eye with the forehead, suggests that Piero della Francesca opted to show Federico's profile not exactly as seen from the side, but slightly from behind. In this way, as spectators, we literally get a small glimpse of the extended eye field that Federico would have accessed when his gaze shifted towards the nasal side. Curiously, Piero della Francesca also painted Federico on the reverse side of the painting, in an allegorical depiction showing the duke seated on a palanquin, participating in a triumphant procession. 
Here, Federico is painted from the right, showing the scarred nose bridge and an apparently intact right eye - a detail not yet formally noted..$^{(1)}$

In the portrait attributed to Pedro Berruguete $(1450-1504),{ }^{(2)}$ commissioned ten years later, Federico - besides bearing a few new facial scars - is shown in a true profile view from the side (figure 1b). Here, the ear is further back, and the socket of the eye is placed appropriately behind the brow. Again, the artist gives one an uncanny subliminal glimpse into Federico's vision. The duke tilts the book towards the light while holding it at a distance - the sign of approaching presbyopia, perhaps - and with his head facing the book; nevertheless, he seems to be reading from the right page without having to turn his head towards the right. This gives him a completely normal appearance; but knowing that his right eye was missing, it leaves us with the impression that this extended visual access was quite useful in his daily life.

\section{The 'advantages' of monocular vision}

Despite losing his binocular vision, the duke must have retained other depth cues, such as motion parallax. Ocular parallax between near and distant objects should feature as a primary, omnipresent parallax depth cue that heightens depth perception whenever we make eye movements. This is, in a sense, free depth information, since it is there whenever one makes a saccade. Whether ocular parallax serves as a significant depth cue in everyday vision remains to be scientifically verified. Enucleation of one eye, robbing the visual system completely of binocular input, may pave the way for heightened sensitivity to ocular parallax cues derived from the remaining eye.

Another possible advantage of the monocular warrior is that in all likelihood the ocular egocenter could be expected to migrate towards the intact eye. It has been observed to migrate by about $75 \%$ of the distance towards the remaining eye in some case studies by Moidell et al (1988). Interestingly, inexperienced gun users and amateur archers tend to close one eye to improve their aim imposing a temporary enucleation to themselves. That the lack of rivalry improves the ability to aim, should not really come as a surprise. Binocular rivalry, occurring at roughly once every two seconds, could hinder the ability to take a steady aim at a distant target. Among professionals, there are many rules of thumb for unlearning the urge to close one eye. What we casually observe in professionals as they take aim with both eyes open is that they no longer foveate the weapon in relation to the target; they have internalized their weapon to the extent where they need to look at only the target (in sport, the adage of keeping your eye on the ball!). The motorsomatic cues from holding the weapon are likely sufficient in helping them take and maintain proper aim. We would speculatively propose that greater alignment between the cyclopean center and one real eye should improve one's aim while shooting or wielding a sword. Federico could literally use his nose bridge as a sight with which to fix his aim, especially towards foot soldiers if he was mounted on a horse.

${ }^{(1)}$ There may be sound logic in Piero della Francesca's choice to show the right profile of Federico on the reverse side of the painting shown in figure 1a. The portrait forms one half of a diptych, the other panel showing his wife, Battista Sforza, with the couple facing each other in profile. The recto, or reverse side, of the diptych shows the couple again facing each other, each riding on a palanquin. During opening and closing of the diptych, it would be more befitting if the image of Federico did not suddenly switch places with that of Battista, but if each maintained its 'identity', staying on its own side, so to speak.

(2) Most art historians agree in attributing the painting to Pedro Berruguete; nevertheless, others attribute the duke's portrait with his son to Giusto di Gand, known also as Joos van Wassenhove, a Flemish painter who worked in Urbino between 1472-1475 and who collaborated with Pedro Berruguete in Urbino. Still others claim that the author of the portrait is Melozzo da Forli (1438-1494), present in Urbino in those same years, and whose style was influenced by the perspective studies conducted by Piero della Francesca and who influenced the younger Pedro Berruguete (Zocca 1960). In this context it is worth noting that Federico da Montefeltro acted as patron to a wide array of local and foreign artists - testimony that he embraced the arts, culture, and learning. 


\section{Shared visions}

Masamune Date (1567-1636), a renowned samurai warrior from Japan, also lost his right eye. In his case, a spell of childhood smallpox was to blame. According to popular folklore, he also opted for an act of self-mutilation in order to enable himself to rise through the ranks of the samurai community. Purportedly, he plucked his own dysfunctional right eyeball out of its socket immediately after a superior voiced a concern that his enemies may defeat him by grabbing hold of it. Forensic analysis of his skull did not yield any evidence to support this account (Suzuki 1998); hence the story is most likely merely a popular fabrication. Masamune apparently had superior martial skills, had no difficulty in wielding his weapons, but never opted for any surgical alteration of his nose bridge. Mapp and Ono (1986) compare eye fields and nasal protrusion of Caucasian and Far East Asian subjects. While the lower bridge of typical Far East Asian noses intrudes much less into the eye field when they look towards the nasal side, the eye field in itself is not as wide for Caucasians when they look straight ahead - a feature ascribed to optical differences caused by the physical structure of the eye between the different subject groups. So Masamune Date did not need to adjust his nose. After a failed attempt by his mother to poison him (she was hoping to give Masamune's younger brother a chance to advance his military career), his sibling met his end in a brutal assassination. Federico's potentially rivalrous sibling followed a similar trajectory (Benzoni 1995). Both warriors were feared and revered, both had long careers compared with their rivals and amassed huge personal fortunes, and both were known for being as well cultured and well versed in the classics as they were familiar with the battlefield. Federico had himself painted in profile, deliberately confronting the orthogonal cut to his nose. Masamune was depicted with his deformity, the local custom in Japan at the time being that lower-ranking figures were shown in profile, while frontal views were reserved for those of higher status.

Both men often went against the powers of the day, showing great mercy to those who surrendered promptly, while obliterating any resistance. Their greatest victories were not in physical combat, but through diplomacy. Masamune actively encouraged foreigners to adopt Japan as their home, hailing a very prosperous new era in the otherwise closed history of the islands, and he masterminded the first Japanese convoy to circle the globe. In Italy, Federico turned Urbino into an important center of learning and art patronage. Ironically, he died of fever in Ferrara in 1482, while he was defending the city against the armies of Venicearmies sent by the very pope he defended for the major duration of his successful career. Two hundred years after his death, the envoy of another one-eyed warrior, Masamune, had an audience with the pope in Rome.

Considering the achievements of these two illustrious figures, it seems as if losing a right eye endows one with a much enlarged vision of the world. Or is it just that one finally can see the parallax?

\section{References}

Baldi B, 1824 Vita e fatti di Federigo di Montefeltro, duca di Urbino, istoria di Bernardino Baldi, estratta da ms. inedito della Biblioteca Albani, e corredata di osservazioni del sig. avv. Francesco Zuccardi (Rome: Salvioni)

Benzoni G, 1995 "Federico da Montefeltro, duca di Urbino", in Dizionario biografico degli italiani volume 45 (Rome: Istituto dell'Enciclopedia Italiana) pp 722-743

Brewster D, 1844 "On the law of visible position in single and binocular vision and on the representation of solid figures by the union of dissimilar plane pictures on the retina" Transactions of the Royal Society of Edinburgh 15 349-368

Mapp A P, Ono H, 1986 "The rhino-optical phenomenon: ocular parallax and the visible field beyond the nose" Vision Research 26 1163-1165

Moidell B, Steinbach M J, Ono H, 1988 "Egocenter location in children enucleated at an early age" Investigative Opthalmology \& Visual Science 29 1348-1351 
Roeck B, Tönnesmann A, 2005 Die Nase Italiens: Federico da Montefeltro, Herzog von Urbino (Berlin: Wagenbach)

Suzuki H, 1998 Hone ga kataru nihonshi [Japanese history as told by skeletons] (Tokyo: Gakuseisha) Wade N J (Ed.), 1983 Brewster and Wheatstone on Vision (London: Academic Press)

Zocca E, 1960 "AMBROSI, Melozzo degli, detto Melozzo da Forli” Dizionario biografico degli italiani volume 2 (Rome: Istituto dell'Enciclopedia Italiana) pp 724-725 